\title{
Ensembles of Classifiers for Handwritten Word Recognition Specialized on Individual Handwriting Style
}

\author{
Simon Günter and Horst Bunke \\ Department of Computer Science, University of Bern \\ Neubrückstrasse 10, CH-3012 Bern, Switzerland \\ \{sguenter, bunke\}@iam. unibe.ch
}

\begin{abstract}
The study of multiple classifier systems has become an area of intensive research in pattern recognition recently. Also in handwriting recognition, systems combining several classifiers have been investigated. Recently, new methods for the generation of multiple classifier systems, called ensemble methods, have been proposed in the field of machine learning, which generate an ensemble of classifiers from a single classifier automatically. In this paper a new ensemble method is proposed. It is characterized by training each individual classifier on a particular writing style. The new ensemble method is tested on two large scale handwritten word recognition tasks.
\end{abstract}

Keywords: handwritten word recognition, ensemble method, writing style, hidden Markov model (HMM)

\section{Introduction}

The field of off-line handwriting recognition has been a topic of intensive research for many years. First only the recognition of isolated handwritten characters was investigated [29], but later whole words [28] were addressed. Most of the systems reported in the literature until today consider constrained recognition problems based on vocabularies from specific domains, e.g. the recognition of handwritten check amounts [15] or postal addresses [17]. Free handwriting recognition, without domain specific constraints and large vocabularies, was addressed only recently in a few papers $[18,24]$. The recognition rate of such systems is still low, and there is a need to improve it.

The combination of multiple classifiers was shown to be suitable for improving the recognition performance in difficult classification problems [20,30]. Also in handwriting recognition, classifier combination has been applied. Examples are given in $[2,22,31]$. Recently methods for the generation of multiple classifier systems, called ensemble methods, have been proposed in the field of machine learning, which generate an ensemble of classifiers from a single classifier [5] automatically. Given a single classifier, the base classifier, a set of classifiers can be generated by changing the training set [3], the input features [14], the input data by injecting randomness [7], or the parameters and the architecture 
Table 1. The new ensemble method

Input: The original training set $T$, base classifier $C$, and the desired number of classifiers, $n$

Output: $n$ classifiers.

A clustering algorithm is applied on $T$, resulting in $n$ clusters ;

for $(\mathrm{i}:=1 ; \mathrm{i}<=\mathrm{n} ; \mathrm{i}++)$

$T_{i}$ contains all elements of the $i$-th cluster;

classifier $C$ is trained on $T_{i}$, resulting in classifier $C_{i}$;

return $C_{1}, \ldots, C_{n}$;

of the classifier [26]. Another possibility is to change the classification task from a multi-class to many two-class problems [6]. Examples of widely used methods that change the training set are Bagging [3] and AdaBoost [8]. Random subspace method [14] is a well-known approach based on changing the input features. A summary of ensemble methods is provided in [5].

Although the popularity of multiple classifier systems in handwritten recognition has grown significantly, not much work on the use of ensemble methods has been reported in the literature. An exception is [25], but this paper addresses only the classification of isolated characters, while the focus of the present paper is on the recognition of cursive words. Some papers of the authors addressed the use of ensemble methods for handwritten word recognition (e.g. [10, 12]).

In this paper we propose a new ensemble method. This ensemble method specializes the individual classifiers on different writing styles. In Section 2 the new ensemble method is described in detail. Section 3 contains information about the handwritten word recognizer, which is used as the base classifier in the experiments. Those experiments are then discussed in Section 4 and, finally, conclusions are drawn in Section 5.

\section{New Ensemble Creation Method}

In this section the new ensemble method is introduced. The basic algorithm of the ensemble method is given in Table 1. First, the training set is divided into $n$ disjoint subsets, using a clustering algorithm. Then each classifier is trained on the elements of a cluster. This results in $n$ different classifiers. There are two key parameters of the ensemble method: the clustering algorithm and the number of clusters, $n$. The novel idea of the paper is that we want to specialize the classifiers on different writing styles. The intuitive motivation of this idea is that the models of the words may not be able to handle too many different writing styles and so inadequately trained models could be produced. By reducing the number of writing styles, i.e. by focusing on individual clusters, the quality of the models may increase. This is in contrast to most classical ensemble methods where one selects words randomly for the training of the classifiers, like Bagging [3], or where the selection is done according to how difficult it is for the system to recognize a particular word, like AdaBoost [8]. 
The clustering algorithm should produce clusters containing words of similar writing style. In order to execute this clustering, some features that characterize a particular handwriting style must be defined. As we are using the data of the IAM database [23], we always have complete pages of handwritten text at our disposal (compare Section 4). Therefore the features used in this paper are extracted from a whole page, and all words of the page belong to the same cluster. This means that in fact not the words, but the pages are clustered. The features used for the clustering are the following:

- Words per component (WPC): The average number of words per connected component is calculated over the whole page. All components with a bounding box smaller than a given threshold (500 pixels in the experiments reported in Section 4) are ignored, as they are likely to be noise or parts of characters which are always separated from the main part of the character, e.g. the dot of the character "i". Words consisting of single characters, like punctuation, and numbers are also not considered. A value of one of this feature corresponds to a complete cursive handwriting, i.e. the case where one connected component always represents exactly one word. By contrast, words consisting of isolated hand-printed characters with a number of characters of $n$ have the value $\frac{1}{n}$ for this feature. As the clustering procedure is applied on the training set and as the ground truth of each page is known, the feature is simply calculated by dividing the number of words by the number of connected components.

- Character width (CW): The average width of the characters is calculated over the whole page. Again single characters are not considered and components with a bounding boxes smaller than a threshold are ignored. To calculate the width of the characters the bounding boxes of the words are used and so the white spaces between the words are not taken into account. To calculate the values of this feature the lines of each page are segmented into words using the procedure presented in [33]. The character width is then calculated as the sum of the lengths of the words of a page divided by the number of characters.

There are three possible feature sets: Both features alone (WPC,CW) and the feature set consisting of both features (WPC/CW). In previous experiments the feature set CW did not produce good results, so only the other two feature sets will be used in the experiments of this paper.

Please note that not all features that are meaningful can be used in the actual system. For example, slant is a very powerful feature for humans to discriminate different handwriting styles. Yet in our system, slant normalization is done, which makes the slant nearly vertical for all words.

For clustering the words, the $k$-means clustering algorithm [16] with the features described above was executed, where the features were first linearly normalized so that the mean of the features was 0 and the standard deviation was 1 .

When increasing the number of clusters, $n$, which is equal to the number of produced classifiers, the diversity of the writing styles in a cluster decreases and 
better models for those words can be built. On the other hand, a higher number of clusters also means that fewer words are available to train the models, and the parameters of the models are poorly estimated when the number of clusters is too high. The optimal number of clusters, $n$, is apriori unknown and must be empirically determined.

\section{Handwritten Word Recognizer}

The basic handwritten text recognizer used in the experiments of this paper is similar to the one described in [24]. It follows the classical architecture and consists of three main modules: the preprocessing, where noise reduction and normalization take place, the feature extraction, where the image of a handwritten text is transformed into a sequence of numerical feature vectors, and the recognizer, which converts this sequence of feature vectors into a word class.

The first step in the processing chain, the preprocessing, is mainly concerned with text image normalization. The goal of the different normalization steps is to produce a uniform image of the writing with less variations of the same character or word across different writers. The aim of feature extraction is to derive a sequence of feature vectors which describe the writing in such a way that different characters and words can be distinguished, but avoiding redundant information as much as possible. In the presented system the features are based on geometrical measurements. At the core of the recognition procedure is an HMM classifier. It receives a sequence of feature vectors as input and outputs a word class. In the following these modules are described in greater detail.

\subsection{Preprocessing}

Each person has a different writing style with its own characteristics. This fact makes the recognition task complicated. To reduce variations in the handwritten texts as much as possible, a number of preprocessing operations are applied. The input for these preprocessing operations are images of words extracted from the database described in $[23,33]$. The images were scanned with a resolution of 300 dpi. Please note that the system is optimized for this resolution. In the presented system the following preprocessing steps are carried out:

- Skew Correction: The word is horizontally aligned, i.e. rotated, such that the baseline is parallel to the $x$-axis of the image.

- Slant Correction: Applying a shear transformation, the writing's slant is transformed into an upright position.

- Line Positioning: The word 's total extent in vertical direction is normalized to a standard value. Moreover, applying a vertical scaling operation the location of the upper and lower baseline are adjusted to a standard position.

An example of these normalization operations is shown in Fig. 1. For any further technical details see [24]. Please note that there is no normalization of the stroke width of the handwriting. 

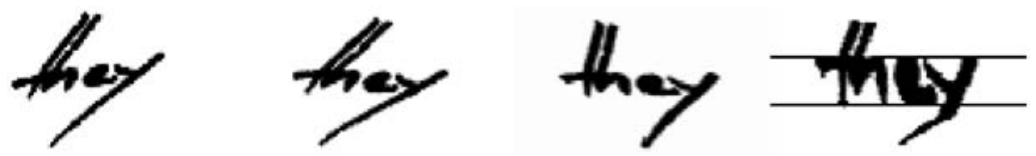

Fig. 1. Preprocessing of the images. From left to right: original, skew corrected, slant corrected and positioned. The two horizontal lines in the right most picture are the two baselines, which are automatically detected

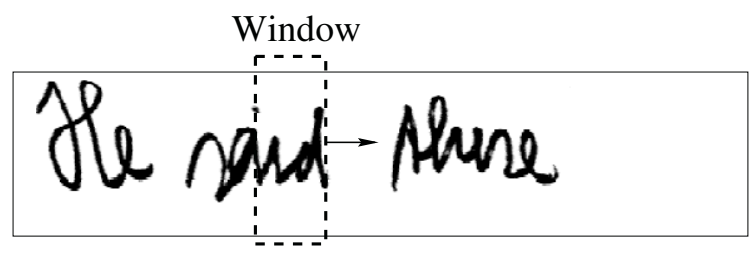

Fig. 2. Illustration of the sliding window technique. A window is moved from left to right and features are calculated for each position of the window. (For graphical representation purposes, the window depicted here is wider than one pixel.)

\subsection{Feature Extraction}

To extract a sequence of feature vectors from a word, a sliding window is used. The width of the window used in the current system is one pixel and its height is equal to the word's height. The window is moved from left to right over each word. (Thus there is no overlap between two consecutive window positions.) Nine geometrical quantities are computed and used as features at each window position. A graphical representation of this sliding window technique is shown in Fig. 2.

The first three features are the weight of the window (i.e. the number of black pixels), its center of gravity, and the second order moment of the window. This set characterizes the window from the global point of view. It includes information about how many pixels in which region of the window are, and how they are distributed. The other features represent additional information about the writing. Features four and five define the position of the upper and the lower contour in the window. The next two features, number six and seven, give the orientation of the upper and the lower contour in the window by the gradient of the contour at the window's position. To compute the contour direction, the windows to the left and to the right of the actual window are used. As feature number eight the number of black-white transitions in vertical direction is used. Finally, feature number nine gives the number of black pixels between the upper and lower contour. Notice that all these features can be easily computed from the binary image of a text line. However, to make the features robust against different writing styles, careful preprocessing, as described in Subsection 3.1, is necessary.

To summarize, the output of the feature extraction phase is a sequence of 9-dimensional feature vectors. For each word to be recognized there exists one 
such vector per pixel along the x-axis, i.e. along the horizontal extension of the considered word.

\subsection{Hidden Markov Models}

Hidden Markov models (HMMs) are widely used in the field of pattern recognition. Their original application was in speech recognition [27]. But because of the similarities between speech and cursive handwriting recognition, HMMs have become very popular in handwriting recognition as well [21].

When using HMMs for a classification problem, an individual HMM is constructed for each pattern class. For each observation sequence, i.e. for each sequence of feature vectors, the likelihood that this sequence was produced by an HMM of a class can be calculated. The class whose HMM achieves the highest likelihood is considered as the class that produced the actual sequence of observations. For further details and an introduction to HMMs see [27], for example.

In word recognition systems with a small vocabulary, it is possible to build an individual HMM for each word. But for large vocabularies this method doesn't work anymore because of the lack of enough training data. Therefore, in our system an HMM is built for each character. The use of character models allows us to share training data. Each instance of a character in the training set has an impact on the training and leads to a better parameter estimation.

To achieve high recognition rates, the character HMMs have to be fitted to the problem. In particular the number of states, the possible transitions and the type of the output probability distributions have to be chosen.

The number of states of the HMMs were optimized by the Quantile method [34] for each character individually. As a result, each individual character model has a different number of states. Because of the left to right direction of writing, a linear transition structure has been chosen for the character models. From each state only the same or the succeeding state can be reached. Because of the continuous nature of the features, probability distributions for the features are used. Each feature has its own probability distribution and the likelihood of an observation in a state is the product of the likelihoods calculated for all features. This separation of the elements of the feature vector reduces the number of free parameters, because no covariance terms must be calculated. The probability distribution of all states and features are assumed to be Gaussian mixtures. The training method of the classifier was optimized on a validation set, using an optimization strategy described in [9].

To model entire words, the character models are concatenated with each other. Thus a recognition network is obtained (see Fig. 3). Note that this network doesn't include any contextual knowledge on the character level, i.e., the model of a character is independent of its left and right neighbor. In the network the best path is found with the Viterbi algorithm [27]. It corresponds to the desired recognition result, i.e., the best path represents the sequence of characters with maximum probability, given the image of the input word. The architecture shown in Fig. 3 makes it possible to avoid the difficult task of segmenting a word into 


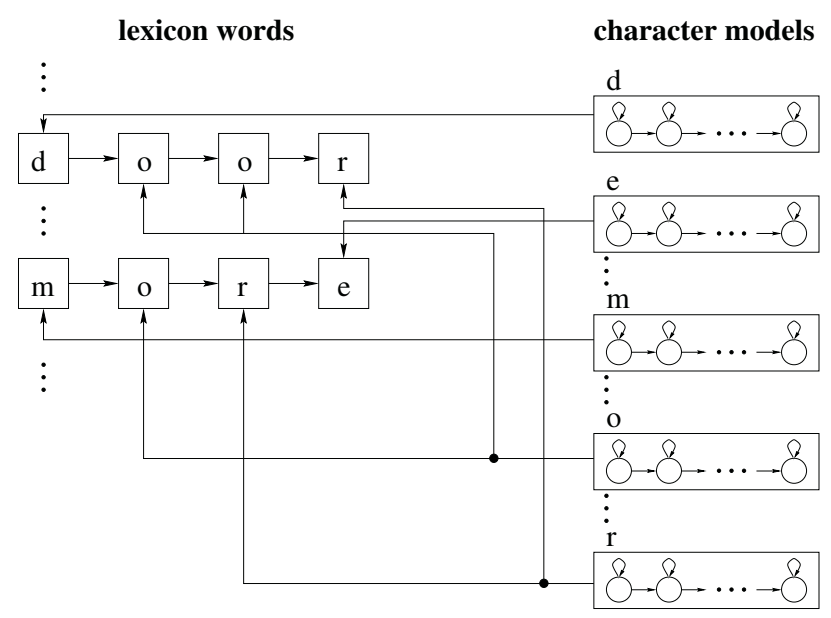

Fig. 3. Concatenation of character models yields the word models

individual characters. More details of the handwritten text recognizer can be found in [24].

The implementation of the system is based on the Hidden Markov Model Toolkit (HTK), which was originally developed for speech recognition [32]. This software tool employs the Baum-Welch algorithm for training and the Viterbi algorithm for recognition [27]. The output of the HMM classifier is the word with the highest rank among all word models together with its score value.

\section{Experiments}

For isolated character and digit recognition, a number of commonly used databases exist. However, for the task considered in this paper, there exists only one suitable database to the knowledge of the authors, holding a sufficiently large number of words produced by different writers, the IAM database [23]. Consequently this database was used in the experiments. An important property of the IAM database is that it consists of whole pages of handwriting, each typically holding more than 50 words. This allows to extract very stable values of the features mentioned in Section 2.

Two different handwriting recognition tasks were considered: A writer dependent and a writer independent task. In the first task a data set of 18,920 words was used. A total of 116 writers contributed to this set. The set of 18,920 words was three times randomly split in a training set of 17,920 words and a test set of 1'000 words. Please note that that the writers of the training words are the same as the writers of the test words which implies that the experimental setup is writer dependent. The recognition rate was calculated by averaging over the system's recognition rates obtained for each of the three splits. In the second task the whole data set for the first task is used as training set. An additional 
data set of 3,264 words, contributed by 37 writers, was used as test set. The set of writers of the training set and the set of writers of the test set are disjoint, so the experimental setup is writer independent. It should be noted that the first task was also used to optimize the number of states of the HMMs and to optimize the training method of the base classifier. As these optimization steps are applied to the base classifier rather than to the ensemble they are not expected to favor the ensemble over the base classifier.

To combine the individual classifiers of the ensembles the following combination schemes were applied:

1. Maximum score (score): Only the top choice of each classifier is considered. The result of the classifiers with the highest score value is used as the result of the classifier ensemble.

2. Voting scheme (voting): Again, only the top choice of each classifier is considered. The word class that is most often on the first rank is the output of the combined classifier. Ties are broken by means of the average rule (ave), maximum rule $(\max )$, the minimum rule $(\mathrm{min})$ and the median rule $(\mathrm{med})$, which are only applied to the competing word classes. The four voting rules mentioned above decide for the word class which has the highest average, maximum, minimum or median score, respectively.

3. Special combination scheme for HMM-based recognizers (special): This combination was introduced in [11]. It integrates all HMMs of the different classifiers that correspond to the same character into a single HMM.

The results obtained for the first task are shown in Table 2. The recognition rate of the base classifier was $80.03 \%$. The columns feature sets and $n$ denote the underlying feature set and the number of classifiers, respectively. The best result of each feature set is marked in bold. Please note that all four versions of the voting scheme are the same for $n=2$ and $n=3$. Therefore the results of only one version is shown. In addition the score combination scheme is the same as the voting scheme for $n=2$, so only the results of the score scheme is depicted.

The results of the special combination scheme are clearly superior to those of the other schemes. In 7 out of 8 cases the best recognition rate was obtained using this scheme. Both the special and the score combination schemes were consistently superior to the voting scheme. Using the special scheme the performance of the base classifier was increased by $2.84 \%$ for $\{\mathrm{WPC}\}$ and $3.13 \%$ for $\{\mathrm{WPC}, \mathrm{CW}\}$. It seems that both feature sets are quite suited for the new ensemble method. The optimal number of classifiers is 3 for the considered application. Please note that this number is rather small. The reason for the small optimal number of $n$ is that the base classifier contains a high number of free parameters which are poorly estimated when using more than four clusters. The increase of the performance is not based on the higher number of HMM parameters, as the number of parameters of the base classifier was (implicitly) optimized by optimizing the number of states and the number of Gaussians per Gaussian mixture. 
Table 2. Results of the new ensemble method for the first task. The performance of the base classifier is $80.03 \%$. The number of clusters is given in the column $n$

\begin{tabular}{|l|l|c|c|}
\hline & & \multicolumn{2}{|c|}{ feature sets } \\
\hline$n$ & lombination & $\{$ WPC $\}$ & $\{$ WPC,CW \\
\hline \hline 2 & score & $81.7 \%$ & $81.73 \%$ \\
\hline 2 & special & $82.4 \%$ & $82 \%$ \\
\hline \hline 3 & score & $82.6 \%$ & $82.63 \%$ \\
\hline 3 & special & $82.56 \%$ & $\mathbf{8 3 . 1 7} \%$ \\
\hline 3 & voting & $82.03 \%$ & $81.83 \%$ \\
\hline \hline 4 & score & $82.63 \%$ & $82.1 \%$ \\
\hline 4 & special & $\mathbf{8 2 . 8 7} \%$ & $82.87 \%$ \\
\hline 4 & voting ave & $81.73 \%$ & $79.97 \%$ \\
\hline 4 & voting max & $81.63 \%$ & $80.06 \%$ \\
\hline 4 & voting min & $81.63 \%$ & $79.67 \%$ \\
\hline 4 & voting med & $81.77 \%$ & $79.67 \%$ \\
\hline 5 & score & $81.8 \%$ & $82.33 \%$ \\
\hline 5 & special & $82.43 \%$ & $82.67 \%$ \\
\hline 5 & voting ave & $80.5 \%$ & $80.57 \%$ \\
\hline 5 & voting max & $80.6 \%$ & $80.6 \%$ \\
\hline 5 & voting min & $80.47 \%$ & $80.47 \%$ \\
\hline 5 & voting med & $80.47 \%$ & $80.47 \%$ \\
\hline
\end{tabular}

Table 3. Results of the ensemble method for the second task. The performance of the base classifier is $80.48 \%$. The number of clusters is given in the column $\mathrm{cn}$

\begin{tabular}{|c|c|c|}
\hline feature set & cn & performance \\
\hline$\{\mathrm{WPC}\}$ & 3 & $80.48 \%$ \\
\hline$\{\mathrm{WPC}\}$ & 4 & $80.73 \%$ \\
\hline$\{\mathrm{WPC}, \mathrm{CW}\}$ & 3 & $81.07 \%$ \\
\hline$\{\mathrm{WPC}, \mathrm{CW}\}$ & 4 & $80.64 \%$ \\
\hline
\end{tabular}

For the second task only the parameter values which produced good results in the first task were used. Therefore only cases where we have 3 or 4 classifiers in the ensembles were tested and the ensemble were always combined by the special scheme. Table 3 shows the results of the ensemble method for the second task. The recognition rate of the base classifier which is trained on the full training set was $80.48 \%$.

The performance of the ensemble method was always better or the same as the performance of the base classifier. The obtained increases in the recognition rate are rather low when compared to the results in Table 2. Please note that for the best configuration in Table 2 , i.e. 3 clusters and feature set $\{\mathrm{WPC}, \mathrm{CW}\}$, also the best result in Table 3 was achieved. With these parameter values a performance increase of $0.59 \%$ in respect of the base classifier has been obtained. This increase is statistically significant using a significance level of $15 \%{ }^{1}$.

\footnotetext{
${ }^{1}$ Where the null-hypothesis is that both the base classifier and the ensemble have the same performance.
} 
The good results of the first and the modest results of the second task indicate that the approach works best when the writer of the test word already contributed data to the training set, i.e. the system already "knows" the writing style of the test word.

\section{Conclusions}

A new ensemble method was introduced where each classifier is specialized on a different writing style. Two different handwritten word recognition tasks were considered. In the first task a writer depended setup was used where the writers of the tested words also contributed words to the training set. The second task is writer independent where the writers of the training words did not contribute words to the training set. The ensemble method achieved very good results for the writer dependent setup. The performance of the base classifier was increased up to $3.13 \%$. The performance gain for the writer independent setup was modest, but still the ensemble method did always better than the base classifier or had the same performance. In the best case an improvement of $0.59 \%$ was achieved. It seems that the new ensemble method is particually good for writer dependent setups.

Future research will focus on the use of other feature sets for the clustering of the training elements. For example some of the features introduced in [13] may be used. Additionally, the use of different values for the HMM and training parameters of the individual classifiers may be investigated. Such parameters are for example the number of states and the training method. The values of those parameters were optimized for the full training set and may be suboptimal when using smaller training sets.

\section{Acknowledgment}

This research was supported by the Swiss National Science Foundation (Nr. 20-52087.97). The authors thank Dr. Urs-Victor Marti for providing the handwritten word recognizer and Matthias Zimmermann for the segmentation of a part of the IAM database. Additional funding was provided by the Swiss National Science Foundation NCCR program "Interactive Multimodal Information Management (IM)2" in the Individual Project "Scene Analysis".

\section{References}

1. Proc of the 7th Int. Conf. on Document Analysis and Recognition, Edinburgh, Scotland, 2003.

2. A. Brakensiek, J. Rottland, A. Kosmala, and G. Rigoll. Off-line handwriting recognition using various hybrid modeling techniques and character n-grams. In 7th International Workshop on Frontiers in Handwritten Recognition, pages 343-352, 2000 .

3. Leo Breiman. Bagging predictors. Machine Learning, (2):123-140, 1996. 
4. H. Bunke and P. Wang, editors. Handbook of Character Recognition and Document Image Analysis. World Scientific, 1997.

5. T. G. Dietterich. Ensemble methods in machine learning. In [19], pages 1-15.

6. T. G. Dietterich and G. Bakiri. Solving multiclass learning problems via errorcorrecting output codes. Journal of Artifical Intelligence Research, 2:263-286, 1995.

7. T.G. Dietterich and E.B. Kong. Machine learning bias, statistical bias, and statistical variance of decision tree algorithms. Technical report, Departement of Computer Science, Oregon State University, 1995.

8. Yoav Freund and Robert E. Schapire. A decision-theoretic generalisation of online learning and an application to boosting. Journal of Computer and Systems Sciences, 55(1):119-139, 1997.

9. S. Günter and H. Bunke. Optimizing the number of states, training iterations and Gaussians in an HMM-based handwritten word recognizer. In [1], volume 1, pages $472-476$.

10. S. Günter and H. Bunke. Creation of classifier ensembles for handwritten word recognition using feature selection algorithms. In Proc. of the 8th International Conference on Frontiers in Handwriting Recognition, pages 183-188, Niagara-onthe-Lake, Canada, 2002.

11. S. Günter and H. Bunke. A new combination scheme for HMM-based classifiers and its application to handwriting recognition. In 16th International Conference on Pattern Recognition, volume 2, pages 332-337, Quebec, Canada, 2002.

12. S. Günter and H. Bunke. Ensembles of classifiers for handwritten word recognition. Int. Journal on Document Analysis and Recognition, 5(4):224 - 232, 2003.

13. C. Hertel and H. Bunke. A set of novel features for writer identification. In Proc. of the 4th Int. Conference on Audio-and Video-Based Biometric Person Authentication AVBPA, pages 679 - 687, Guildford, UK, 2003.

14. T. K. Ho. The random subspace method for constructing decision forests. IEEE Transactions on Pattern Analysis and Machine Intelligence, 20(8):832-844, 1998.

15. S. Impedovo, P. Wang, and H. Bunke, editors. Automatic Bankcheck Processing. World Scientific Publ. Co, Singapore, 1997.

16. A. Jain, M. Murty, and P. Flynn. Data clustering: A survey. ACM Computing Survey, 31:264-323, 1999.

17. A. Kaltenmeier, T. Caesar, J.M. Gloger, and E. Mandler. Sophisticated topology of hidden Markov models for cursive script recognition. In Proc. of the 2nd Int. Conf. on Document Analysis and Recognition, Tsukuba Science City, Japan, pages 139-142, 1993.

18. G. Kim, V. Govindaraju, and S.N. Srihari. Architecture for handwritten text recognition systems. In S.-W. Lee, editor, Advances in Handwriting Recognition, pages 163-172. World Scientific Publ. Co., 1999.

19. J. Kittler and F. Roli, editors. First International Workshop on Multiple Classifier Systems, Cagliari, Italy, 2000. Springer.

20. J. Kittler and F. Roli, editors. Third International Workshop on Multiple Classifier Systems, Cagliari, Italy, 2002. Springer.

21. A. Kundu. Handwritten word recognition using hidden Markov model. In [4], pages $157-182$.

22. D. Lee and S. Srihari. Handprinted digit recognition: A comparison of algorithms. In Third International Workshop on Frontiers in Handwriting Recognition, pages 153-162, 1993.

23. U. Marti and H. Bunke. The IAM-database: An English sentence database for offline handwriting recognition. Int. Journal of Document Analysis and Recognition, 5:39-46, 2002. 
24. U.-V. Marti and H. Bunke. Using a statistical language model to improve the performance of an HMM-based cursive handwriting recognition system. Int. Journal of Pattern Recognition and Art. Intelligence, 15:65-90, 2001.

25. H. Nishimura, M. Kobayashi, M. Maruyama, and Y. Nakano. Off-line character recognition using HMM by multiple directional feature extraction and voting with bagging algorithm. In 5th International Conference on Document Analysis and Recognition, pages 49-52, Bangalore, India, 1999.

26. D. Partridge and W. B. Yates. Engineering multiversion neural-net systems. Neural Computation, 8(4):869-893, 1996.

27. L. Rabiner and B.-H. Juang. Fundamentals of Speech Recognition. Prentice Hall, 1993.

28. J.-C. Simon. Off-line cursive word recognition. Special Issue of Proc. of the IEEE, 80(7):1150-1161, July 1992.

29. C.Y. Suen, C. Nadal, R. Legault, T.A. Mai, and L. Lam. Computer recognition of unconstrained handwritten numerals. Special Issue of Proc. of the IEEE, 80(7):1162-1180, 1992.

30. T. Windeatt and F. Roli, editors. 4th Int. Workshop on Multiple Classifier Systems, Guildford, United Kingdom, 2003. Springer.

31. L. Xu, A. Krzyzak, and C. Suen. Methods of combining multiple classifiers and their applications to handwriting recognition. IEEE Transactions on Systems, Man and Cybernetics, 22(3):418-435, 1992.

32. S. J. Young, J. Jansen, J. J. Odell, D. Ollason, and P. C. Woodland. The HTK Hidden Markov Model Toolkit Book. Entropic Cambridge Research Laboratory, http://htk.eng.cam.ac.uk/, 1995.

33. M. Zimmermann and H. Bunke. Automatic segmentation of the IAM off-line database for handwritten English text. In Proc. of 16th Int. Conference on Pattern Recognition, volume 4, pages 35-39, Quebec, Canada, 2002.

34. M. Zimmermann and H. Bunke. Automatic segmentation of the IAM off-line database for handwritten English text. In Proc. of the 16th Int. Conference on Pattern Recognition, volume 4, pages 35-39, Quebec, Canada, 2002. 